\title{
ARRHYTHMIAS
}

\section{Long working hours and risk of atrial fibrillation}

Working long hours is associated with an increased risk of developing atrial fibrillation (AF), according to a new study published in European Heart Journal. Whether the relationship is causal remains to be determined.

Members of the IPD-Work Consortium conducted a prospective, multicohort study involving 85,494 individuals (mean age 43.4 years; $35 \%$ men) with no recorded $\mathrm{AF}$ at baseline. Overall, $5.2 \%$ of participants were working 'long' hours ( $\geq 55 \mathrm{~h}$ per week), and $62.5 \%$ were working 'standard' hours $(35-40 \mathrm{~h}$ per week). During a 10-year follow-up, 1,061 new cases of AF occurred (10-year cumulative incidence 12.4 per 1,000$)$. After adjustment for age, sex, and socioeconomic status, individuals working long hours had a higher risk of incident AF than those working standard hours (HR 1.42, $95 \% \mathrm{Cl} 1.13-1.80, P=0.003)$. Of note, the presence of obesity, smoking, physical inactivity, and high alcohol consumption was higher among individuals with long working hours than in the group working standard hours. Nevertheless, further adjustment for these potential confounding factors had only a marginal effect on the increased risk of AF.

"These findings suggest that long working hours is a risk factor for AF," conclude the investigators. "Further research is needed to determine mechanisms underlying the link." In an editorial that accompanied the study report, Bakhtawar Mahmoodi and Lucas Boersma suggest that "changes in autonomic tone and increasing atrial fibrosis" might connect long working hours to $A F$, but evidence is lacking. Mahmoodi and Boersma also highlight some potentially relevant confounders that were not explored in the analyses, including "the types of jobs (e.g. office vs construction work) and irregular working hours including night shifts".

\section{Gregory B. Lim}

ORIGINAL ARTICLE Kivimäki, M. et al. Long working hours as a risk factor for atrial fibrillation: a multi-cohort study. Eur. Heart J. http://dx.doi.org/10.1093/eurheartj/ehx324 (2017) 